\title{
Significance of Cellular Fatty Acids and Sugars in Defining the Genus Porphyromonas
}

\author{
I. BRONDZ, ${ }^{1 *}$ J. CARLSSON,${ }^{2}$ M. SJÖSTRÖM,${ }^{3}$ AND G. SUNDQVIST ${ }^{4}$ \\ Research Department, National Institute of Occupational Health, S-900 06 Umeå ${ }^{1}$ and Departments of Oral \\ Microbiology ${ }^{2}$ and Endodontics, ${ }^{4}$ and Research Group for Chemometrics, Department of Chemistry, ${ }^{3}$ \\ University of Umeå, S-901 87 Umeå, Sweden
}

\begin{abstract}
It has recently been proposed that three asaccharolytic species forming pigmented colonies on blood agar should be transferred from the genus Bacteroides to the new genus Porphyromonas. In the taxonomy of the genus Bacteroides cellular fatty acid profiles obtained by gas chromatography and mass spectrometry after methylation and derivatization of whole cells have proved to be useful. In this study cellular fatty acids and sugars were analyzed in strains of Porphyromonas and Bacteroides species, and the resulting multivariate data were investigated by using principal component analysis. This analysis clearly separated the Porphyromonas spp. strains from the strains of Bacteroides fragilis, Bacteroides melaninogenicus, Bacteroides intermedius, and Bacteroides levii. In the description of the genus Porphyromonas the presence of various hydroxylated fatty acids was not discussed. In this study we show that these cellular components are useful taxonomic markers.
\end{abstract}

It has been proposed that three species of asaccharolytic, pigmenting, anaerobic, gram-negative rods (Bacteroides asaccharolyticus, Bacteroides gingivalis, and Bacteroides endodontalis) should be transferred to the genus Porphyromonas as Porphyromonas asaccharolytica, Porphyromonas gingivalis, and Porphyromonas endodontalis, respectively (20).

Cellular fatty acid profiles obtained by gas chromatography and mass spectrometry after methylation and derivatization of whole cells have proved to be useful in the taxonomy of the genus Bacteroides $(9,13,16,19)$. However, the data available on the fatty acid compositions of the lipopolysaccharides of the genus Porphyromonas are not consistent. Mansheim et al. (10) and Koga et al. (7) did not find any hydroxylated fatty acids in the lipopolysaccharide of $P$. gingivalis, while Nair et al. (17), Mashimo et al. (12), and Johne et al. (6) found that hydroxylated acids were abundant.

In this study whole cells of five strains of the genus Porphyromonas and four strains of the genus Bacteroides were examined for eight cellular fatty acids and four sugars. The resulting multivariate data table was investigated by using principal component analysis $(11,23)$ to study the similarity and dissimilarity among the Porphyromonas spp. and Bacteroides spp. strains. The lipopolysaccharide of $P$. gingivalis also was analyzed for fatty acids and sugars.

\section{MATERIAL AND METHODS}

Bacterial strains. The following strains were studied: $P$. gingivalis ATCC $33277^{\mathrm{T}}(\mathrm{T}=$ type strain), 381, and W83, $P$. endodontalis ATCC $35406^{\mathrm{T}}, P$. asaccharolytica ATCC $25260^{\mathrm{T}}$, Bacteroides fragilis ATCC $25285^{\mathrm{T}}$, Bacteroides melaninogenicus ATCC $25845^{\mathrm{T}}$, Bacteroides intermedius ATCC 25261, and Bacteroides levii ATCC $29147^{\mathrm{T}}$. Strains ATCC $33277^{\mathrm{T}}$, ATCC $35406^{\mathrm{T}}$, ATCC $25260^{\mathrm{T}}$, ATCC $25285^{\mathrm{T}}$, ATCC 25845 , ATCC 25261 , and ATCC $29147^{\mathrm{T}}$ were obtained from the American Type Culture Collection, Rockville, Md., and strains 381 and W83 were obtained from T. J. M. van

\footnotetext{
* Corresponding author.
}

Steenbergen, Free University, Amsterdam, The Netherlands.

The strains were grown on the surfaces of blood agar plates at $37^{\circ} \mathrm{C}$ in an anaerobic chamber with an atmosphere containing $5 \%$ carbon dioxide and $10 \%$ hydrogen in nitrogen. The blood agar medium was prepared by the method of Holdeman et al. (5). The defibrinated horse blood in the medium was hemolyzed by freeze-thawing. After 5 days the colonies on the plates were scraped off and freeze-dried.

Reference substances and chemicals. Methyl esters of decanoic, dodecanoic, tetradecanoic, pentadecanoic, hexadecanoic, and octadecanoic acids were obtained from Sigma Chemical Co., St. Louis, Mo. Bacterial acid methyl ester mixture CP (catalog no. 4-7080), gas-liquid chromatography standard mixture GLC 70 (catalog no. 4-7044), American Oil Chemists' Society oil reference mixture RM-1 rapeseed (catalog no. 4-7019), and National Institutes of Health reference mixtures (catalog no. A-NHI-C 4-7010, A-NHI-D 4-7011, and A-NHI-F 4-7013) were obtained from Supelco, Inc., Bellefonte, Pa. 13-Methyltetradecanoic acid and 12-methyltetradecanoic acid were obtained from Larodan Fine Chemicals, Malmö, Sweden. The following sugars were obtained from Sigma Chemical Co.: 2-acetamido-2-deoxy3-O-( $\beta$-D-galactopyranosyl)-D-glucopyranose, methyl-3- $O$-(2-acetamido-2-deoxy- $\beta$-D-glucopyranosyl)- $\beta$-Dgalactopyranoside,2-acetoamido-6- $O$-(2-acetoamido-2-deoxy$\beta$-D-glucopyranosyl)-2-deoxy-D-glucopyranose, rhamnose, fucose, galactose, glucose, and mannose. Methanol p.a. and $n$-hexane p.a. were obtained from E. Merck AG, Darmstadt, Federal Republic of Germany.

Preparation and analysis of sugars and fatty acids of whole bacterial cells. Three separate 1 -mg preparations of freezedried bacteria of each strain were analyzed. Each preparation was added to $1 \mathrm{ml}$ of $2 \mathrm{M}$ hydrochloric acid in anhydrous methanol and incubated for $24 \mathrm{~h}$ at $95^{\circ} \mathrm{C}$. The resulting methanolysate was dried with a stream of nitrogen gas and derivatized in $1 \mathrm{ml}$ of a solution containing 1 part of trifluoroacetic acid anhydride (Fluka Chemie AG, Buchs, Switzerland) and 3 parts of acetonitrile (Rathburn Chemicals, Walkerburn, United Kingdom) (2). After derivatization this solution was diluted with $1.5 \mathrm{ml}$ of acetonitrile before 
TABLE 1. Sugars and fatty acids in members of the genera Porphyromonas and Bacteroides as disclosed by gas chromatography and mass spectrometry after methylation and derivatization of whole cells

\begin{tabular}{|c|c|c|c|c|c|c|c|c|c|c|c|c|c|}
\hline \multirow[b]{2}{*}{ Bacterial strain } & \multicolumn{12}{|c|}{ Relative amt $(\%)$ of ${ }^{u}$ : } & \multirow[b]{2}{*}{$\begin{array}{c}C_{\text {iso1s }}{ }^{\prime} \\
C_{\text {ante15 }}\end{array}$} \\
\hline & $\mathrm{Gal}^{b}$ & Man & Glu & $\underset{\text { GalN }}{\text { GluN }}+$ & $C_{14: 0}$ & $\mathrm{C}_{\mathrm{iso15}}$ & $\mathrm{C}_{\text {ante15 }}$ & $\mathrm{C}_{15: 0}$ & $\mathrm{C}_{3-\mathrm{OH}-\mathrm{isol} 5}$ & $\mathrm{C}_{16: 0}$ & $\mathrm{C}_{3-\mathrm{OH}-16}$ & $\mathrm{C}_{3-\mathrm{OH}-\mathrm{iso17}}$ & \\
\hline P. asaccharolytica ATCC $25260^{\mathrm{T}}$ & 9.4 & 0.1 & 11.9 & 3.2 & 1.5 & 44.7 & 0.1 & 0 & 3.2 & 4.4 & 3.9 & 17.2 & 1 \\
\hline P. endodontalis ATCC $35406^{\mathrm{T}}$ & 8.8 & 1.0 & 7.4 & 4.5 & 5.2 & 43.4 & 0.1 & 0 & 5.0 & 4.8 & 4.5 & 15.4 & 1 \\
\hline$P$. gingivalis ATCC $33277^{\mathrm{T}}$ & 14.7 & 1.8 & 16.0 & 2.6 & 2.4 & 25.3 & 5.7 & 0 & 2.4 & 7.7 & 4.8 & 16.6 & 1 \\
\hline$P$. gingivalis strain 381 & 13.6 & 1.8 & 11.0 & 2.2 & 3.8 & 28.2 & 6.6 & 0 & 1.6 & 10.2 & 4.0 & 16.8 & 1 \\
\hline$P$. gingivalis strain W83 & 14.1 & 1.8 & 18.3 & 11.5 & 1.1 & 20.2 & 4.7 & 0 & 2.1 & 5.2 & 3.7 & 17.3 & 1 \\
\hline B. fragilis ATCC $25285^{\mathrm{T}}$ & 15.5 & 1.0 & 21.9 & 3.3 & 0.1 & 8.4 & 18.7 & 1.0 & 0 & 3.3 & 4.7 & 22.0 & 0 \\
\hline B. melaninogenicus ATCC $25845^{\mathrm{T}}$ & 10.2 & 8.7 & 4.6 & 5.4 & 0.8 & 12.4 & 21.7 & 0 & 0 & 6.1 & 5.9 & 24.2 & 0 \\
\hline B. intermedius ATCC 25261 & 14.1 & 0.9 & 18.8 & 7.7 & 0.5 & 11.1 & 15.7 & 0 & 0 & 3.0 & 3.6 & 24.6 & 0 \\
\hline B. levii ATCC $29147^{\mathrm{T}}$ & 3.0 & 0.1 & 3.2 & 5.3 & 1.8 & 22.6 & 13.7 & 0 & 4.0 & 10.9 & 15.4 & 20.0 & 1 \\
\hline
\end{tabular}

${ }^{a}$ The relative amount of a substance is expressed as the area of its peak in a chromatogram as a percentage of the total area of the peaks of all the substances which were examined. Each value is the mean derived from three independent preparations run in duplicate.

${ }^{b}$ Abbreviations: Gal, galactose; Man, mannose; Glu, glucose; GluN + GalN, glucosamine plus galactosamine; $\mathrm{C}_{14 ; 0}$, tetradecanoic acid; $\mathrm{C}_{\text {iso15, }}$ 13-

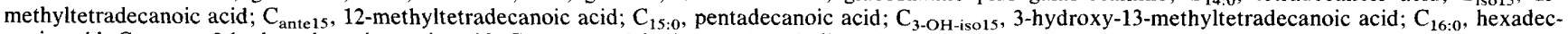
anoic acid; $\mathrm{C}_{3-\mathrm{OH}-16}, 3$-hydroxyhexadecanoic acid; $\mathrm{C}_{3-\mathrm{OH} \text {-isol7 }}$, 3-hydroxy-15-methylhexadecanoic acid.

${ }^{c}$ The value for this character is 1 if the relative amount of $\mathrm{C}_{\text {isols }}$ is greater than the relative amount of $\mathrm{C}_{\text {ante } 15}$ and 0 if the relative amount of $\mathrm{C}_{\mathrm{iso15}}$ is less than the relative amount of $\mathrm{C}_{\text {ante15. }}$.

gas chromatography in a model HP 5890 apparatus equipped with a model HP 5880 integrator (Hewlett-Packard Co., Avondale, $\mathrm{Pa}$.). The chromatograph was equipped with a methyl silicone glass capillary column $(20 \mathrm{~m}$ by $0.3-\mathrm{mm}$ inside diameter; type OV-101; Supelco). A 0.2- $\mu$ l sample was delivered as a splitless injection. The carrier gas was helium, and the flow rate was $2 \mathrm{ml} / \mathrm{min}$. The column temperature was programmed for a $1-\mathrm{min}$ hold at $90^{\circ} \mathrm{C}$ and then an increase to $250^{\circ} \mathrm{C}$ at a rate of $6^{\circ} \mathrm{C} / \mathrm{min}$. The temperature of the injector was $200^{\circ} \mathrm{C}$, and the temperature of the flame ionization detector was $275^{\circ} \mathrm{C}$. The sugars and fatty acids were tentatively identified by comparing their retention times with those of authentic standards. The identities of the sugars and fatty acids were established by direct cochromatography and by gas chromatography-mass spectrometry. Each derivatized preparation was run in duplicate. Thus, the quantification of the recognized substances was based on six runs in the gas chromatograph. The coefficient of variation for these determinations was $3 \%$.

Preparation and analysis of lipopolysaccharide. Lipopolysaccharide of $P$. gingivalis ATCC $33277^{\mathrm{T}}$ was isolated from freeze-dried whole cells by a phenol-water procedure (1) and was extracted three times with hexane at $40^{\circ} \mathrm{C}$. The lipopolysaccharide preparation was divided into three parts, and each part was methanolyzed and derivatized as described by Brondz and Olsen (3). The derivatized samples were analyzed in a model Sigma 3 gas chromatograph (The PerkinElmer Corp., Norwalk, Conn.) equipped with an electronic integrator and a type CP-Sil 5B glass capillary column $(25 \mathrm{~m}$ by $0.22-\mathrm{mm}$ inside diameter; Chromopack, Middelburg, The Netherlands). The conditions used for gas chromatography were the same as those described above for the HewlettPackard instrument.

The instrument used for gas chromatography-mass spectrophotometry was a model 4200 gas chromatograph (Carlo Elba, Milan, Italy) equipped with a model $7072 \mathrm{~F}$ mass spectrometer (Vg Micromass, Cheshire, United Kingdom) and a type 2200 data system (Vg Micromass). The gas chromatograph was equipped with a type CP-Sil 5B glass capillary column as described above, and the conditions for gas chromatography were also the same. The electron impact ionization spectra were recorded under the following conditions: ionization energy, $70 \mathrm{eV}$; ionizing current, 200 $\mu \mathrm{A}$; ion source temperature, $240^{\circ} \mathrm{C}$; and accelerating voltage, $4 \mathrm{kV}$. High-resolution mass spectra were obtained at 70 $\mathrm{eV}$ from a model MS902 double-focus spectrometer connected to a model AE1 computer (Scientific Apparatus, Manchester, United Kingdom).

Statistical methods. In principal component analysis (11, 23) a data matrix $X$ is described by a model. In this matrix the elements consist of the measurements for $M$ variables and $N$ samples. Usually, as in this case, the variables are scaled to unit variance to give all variables initially the same importance. The model is as follows:

$$
x_{i k}=\bar{x}_{k}+\sum_{a=1}^{A} t_{i \mathrm{a}} \mathrm{p}_{\mathrm{ak}}+\mathrm{e}_{\mathrm{ik}}
$$

or in matrix notation

$$
X=1 \bar{x}+T P^{\prime}+E
$$

Here the samples (bacterial strains) are given the index $i$, and the variables (fatty acids and sugars) are given the index $k$. The score vectors $(t)$ are the sample-oriented parameters, and the loading vectors $(p)$ are the variable-related parameters. In the model the first cross-term, $(\mathrm{a}=1) t_{i l} p_{1 k}$, describes the main variance in the data after the variable averages $\bar{x}_{k}$ are subtracted. The next cross-term $(\mathrm{a}=2)$ describes the next greatest variance in the data, etc. The variable loadings give information about which variables contribute to a specific score vector. By plotting the score vectors for the two first cross-terms, the dominating similarity-dissimilarity pattern of the samples can be found.

\section{RESULTS}

The cellular fatty acids and sugars in the members of the genus Porphyromonas are shown in Table 1 . The predominating fatty acids were identified as 13 -methyltetradecanoic $\left(\mathrm{C}_{\text {iso15 }}\right)$ and 3-hydroxy-15-methylhexadecanoic $\left(\mathrm{C}_{3-\mathrm{OH}-\mathrm{iso17}}\right)$ acids. Significant amounts of tetradecanoic $\left(C_{14: 0}\right)$, hexadecanoic $\left(\mathrm{C}_{16: 0}\right)$, 3-hydroxy-13-methyltetradecanoic $\left(\mathrm{C}_{3-\mathrm{OH}-\mathrm{iso15}}\right)$, and 3-hydroxyhexadecanoic $\left(\mathrm{C}_{3-\mathrm{OH}-16}\right)$ acids were also found. 12-Methyltetradecanoic acid $\left(\mathrm{C}_{\text {ante15 }}\right)$ was present in significant amounts in $P$. gingivalis but not in $P$. asaccharolytica or $P$. endodontalis. No pentadecanoic acid 


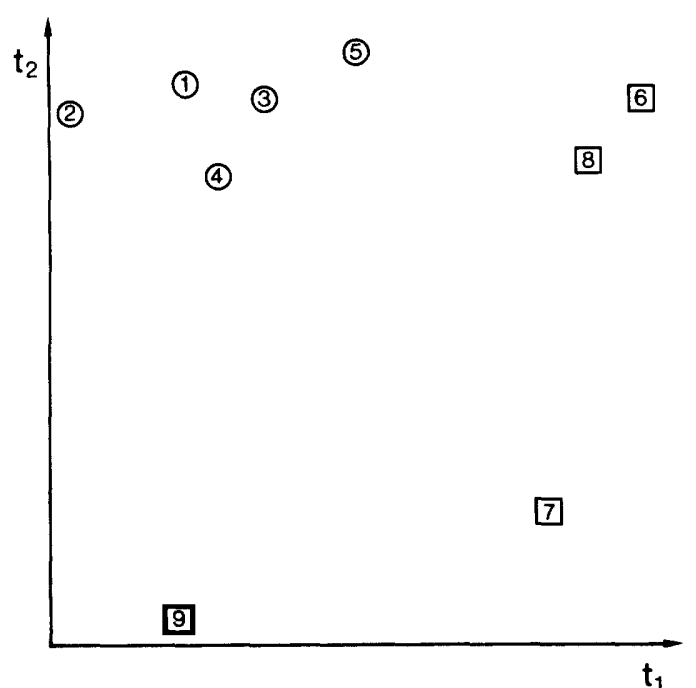

FIG. 1. Sample-oriented (i.e., bacterial strain-oriented) principal component projection $\left(t_{1} / t_{2}\right)$. These two principal components $t_{1}$ and $t_{2}$ describe 33 and $17 \%$, respectively, of the variance of the data in Table 1. Circles indicate strains of Porphyromonas spp., and squares indicate strains of Bacteroides spp., as follows: $1, P$. asaccharolytica ATCC $25260^{\mathrm{T}} ; 2, P$. endodontalis ATCC $35406^{\mathrm{T}} ; 3$, $P$. gingivalis ATCC $33277^{\mathrm{T}} ; 4, P$. gingivalis $381 ; 5, P$. gingivalis W83; 6, B. fragilis ATCC $25285^{\mathrm{T}} ; 7, B$. melaninogenicus ATCC $25845^{\mathrm{T}} ; 8, B$. intermedius ATCC $25261 ; 9, B$. levii ATCC $29147^{\mathrm{T}}$.

$\left(C_{15: 0}\right)$ was detected. Among the cellular sugars galactose, mannose, glucose, and galactosamine, and glucosamine were identified (Table 1).

The cellular fatty acid profiles for $B$. fragilis, $B$. intermedius, and $B$. melaninogenicus were similar to each other, but distinct from the profiles for Porphyromonas species (Table 1). The predominating fatty acids were 12-methyltetradecanoic $\left(\mathrm{C}_{\text {ante15 }}\right)$ and 3-hydroxy-15-methylhexadecanoic $\left(\mathrm{C}_{3-\mathrm{OH}-\mathrm{iso17}}\right)$ acids. Significant amounts of 13-methyltetradecanoic $\left(\mathrm{C}_{\mathrm{iso15}}\right)$, hexadecanoic $\left(\mathrm{C}_{16: 0}\right)$, and 3-hydroxyhexadecanoic $\left(\mathrm{C}_{3-\mathrm{OH}-16}\right)$ acids also were found. No 3-hydroxy-13-methyltetradecanoic acid $\left(\mathrm{C}_{3-\mathrm{OH}-\mathrm{iso15}}\right)$ and only low levels of tetradecanoic acid $\left(\mathrm{C}_{14: 0}\right)$ were detected. Pentadecanoic acid $\left(C_{15: 0}\right)$ was found in $B$. fragilis, but not in $B$. intermedius or $B$. melaninogenicus. All of the fatty acids identified in Porphyromonas species also were found in $B$. levii, but the relative amounts were somewhat different.

The relative amounts of the sugars and fatty acids in $P$. gingivalis lipopolysaccharide (as percentages of the total area of the chromatographic peaks) were as follows: galactose, $14.6 \%$; mannose, $4.7 \%$; glucose, $13.4 \%$; glucosamine plus galactosamine, $19.7 \%$; 3-hydroxy-13-methyltetradecanoic acid $\left(\mathrm{C}_{3-\mathrm{OH} \text {-iso15 }}\right), 5.6 \%$; hexadecanoic acid $\left(\mathrm{C}_{16: 0}\right), 6.6 \%$; 3-hydroxyhexadecanoic acid $\left(\mathrm{C}_{3-\mathrm{OH}-16}\right), 2.9 \%$; and 3-hydroxy-15-methylhexadecanoic acid $\left(\mathrm{C}_{3-\mathrm{OH}-\mathrm{iso17}}\right), 20.5 \%$. These sugars and fatty acids accounted for $88 \%$ of the total chromatographic peak area. Our determination of 2-acetamido-2-deoxy-D-glucoside/galactoside (i.e., glucosamine plus galactosamine) may not be accurate due to simultaneous hydrolysis of the $N$-acetyl group and the glycosyl linkage and a concomitant increase in hydrolysis resistance of the glycosyl linkage.

In the principal component analysis of the data on the cellular fatty acids and sugars of Porphyromonas spp. and Bacteroides spp. strains, the two first principal component score vectors $\left(t_{1}\right.$ and $\left.t_{2}\right)$ are plotted against each other in Fig.

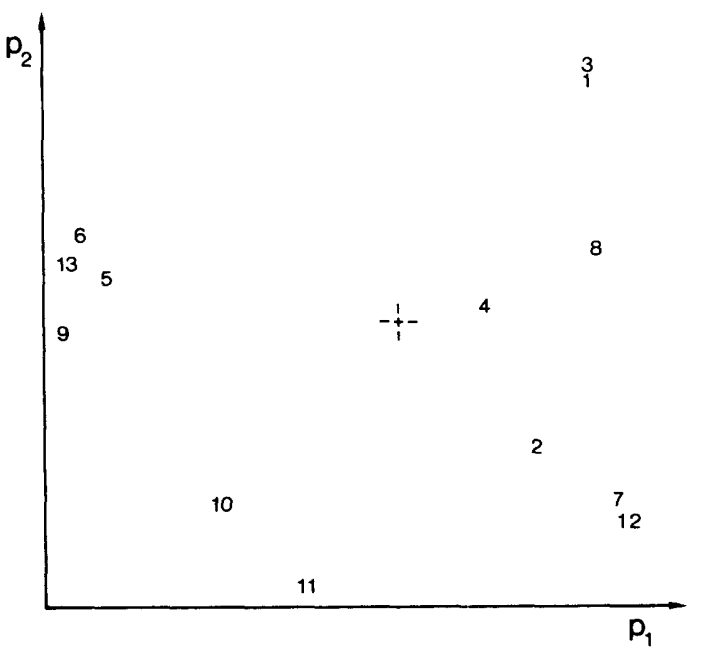

FIG. 2. Variable-oriented (i.e., bacterial character-oriented) principal component projection $\left(p_{1} / p_{2}\right)$. The numbers refer to the following variables: 1 , galactose; 2 , mannose; 3 , glucose; 4 , glucosamine plus galactosamine; 5 , tetradecanoic acid; 6, 13-methyltetradecanoic acid; 7, 12-methyl-tetradecanoic acid; 8, pentadecanoic acid; 9, 3-hydroxy-13-methyltetradecanoic acid; 10, hexadecanoic acid; 11, 3-hydroxyhexadecanoic acid; 12, 3-hydroxy15-methylhexadecanoic acid; 13 , the quotient between the amounts of 13-methyltetradecanoic acid and 12-methyltetradecanoic acid.

1. This sample-oriented (i.e., bacterial strain-oriented) projection describes the two largest variants of the data matrix in Table 1. In this projection the Porphyromonas spp. strains form a homogeneous cluster in the upper left corner of the plot, and the Bacteroides spp. strains are found in the right and lower parts of the plot. Thus, the first score vector $\left(t_{1}\right)$ separates all of the strains of Porphyromonas spp. from the strains of $B$. fragilis, $B$. melaninogenicus, and $B$. intermedius. The second score vector $\left(t_{2}\right)$ separates the Porphyromonas spp. strains from the strains of $B$. melaninogenicus and $B$. levii.

The variables (i.e., bacterial characters [Table 1]) that contribute most to the first score vector $\left(t_{1}\right)$ are tetradecanoic acid $\left(\mathrm{C}_{14: 0}\right)$, 13-methyl-tetradecanoic acid $\left(\mathrm{C}_{\text {iso1s }}\right)$, and 3-hydroxy-13-methyltetradecanoic acid $\left(\mathrm{C}_{3-\mathrm{OH} \text {-iso1s }}\right)$, and those variables that contribute most to the second score vector $\left(t_{2}\right)$ are galactose, mannose, glucose, 12-methyltetradecanoic acid $\left(\mathrm{C}_{\text {ante } 15}\right)$, hexadecanoic acid $\left(\mathrm{C}_{16: 0}\right)$, 3-hydroxyhexadecanoic acid $\left(\mathrm{C}_{3-\mathrm{OH}-16}\right)$ and 3-hydroxy-15-methylhexadecanoic acid $\left(\mathrm{C}_{3-\mathrm{OH}-\mathrm{iso17}}\right)$ (Fig. 2).

The dissimilarities of the strains of Bacteroides spp. compared with the strains of Porphyromonas spp. were also confirmed by first modeling the Porphyromonas class with a separate principal component model with two cross-terms. The complexity needed to describe the Porphyromonas class was determined with cross-validation (22). The deviations of a bacterial strain from the class-describing plane were then expressed as a residual variance. This variance was then compared by using an $\mathrm{F}$ test with the pooled residual variances for the Porphyromonas spp. class. According to this F test, the strains of Bacteroides spp. fell far outside the 95\% confidence interval of the Porphyromonas class.

\section{DISCUSSION}

The results of this study confirmed the cellular fatty acid profiles described by Lambe et al. (9) and Mayberry et al. 
(13) for $P$. gingivalis (13 strains) and $P$. asaccharolytica (16 strains). The cellular fatty acid profile of $P$. endodontalis has not been reported previously. However, this profile was almost identical to that of $P$. asaccharolytica (Table 1); this finding underlines the close taxonomic relationship between $P$. endodontalis and $P$. asaccharolytica (14). The cellular fatty acid profiles found in this study were similar to those reported by Mayberry et al. (13) for B. fragilis (17 strains), $B$. melaninogenicus ( 9 strains), $B$. intermedius (18 strains), and B. levii (11 strains).

More than $80 \%$ of the fatty acids of the lipopolysaccharide preparations of $P$. gingivalis were hydroxylated. The predominating acid was 3-hydroxy-15-methylhexadecanoic acid $\left(\mathrm{C}_{3-\mathrm{OH} \text {-iso17 }}\right)$. Our preparation did not contain significant levels of branched pentadecanoic acids. This is in accordance with the results of Nair et al. (17), but differs from the findings of Mashimo et al. (12), Millar et al. (15), and Johne et al. (6). Branched pentadecanoic acids are the predominating fatty acids of whole-cell preparations. It is possible that these acids come from some cellular lipid source (18) other than lipopolysaccharide. The main difference between our findings and those of Nair et al. (17) was that we did not identify any branched heptodecanoic acid in our preparation. The lipopolysaccharides of Porphyromonas spp. have been considered to be deficient of 3-deoxy-D-manno-2-octulosonic acid (20), and this component was not detected by our technique. However, recent studies on lipopolysaccharides hydrolyzed under various conditions have clearly demonstrated that 3-deoxy-D-manno-2-octulosonic acid is present in the lipopolysaccharides of Porphyromonas spp. $(4,6,8)$.

The principal component analysis of the data on cellular fatty acids and sugars clearly separated the Porphyromonas spp. strains from the strains of $B$. fragilis, $B$. melaninogenicus, B. intermedius, and B. levii (Fig. 1). However, B. levii was distinct from the other Bacteroides spp. strains (Fig. 1).

The principal component analysis also makes it possible to evaluate the significance of a taxonomic marker. A true taxonomic marker is a character of an organism conveying information which also is contained in numerous other characters of the organism. Such a marker contributes to the separation of bacterial taxa, and when it is excluded from the principal component analysis, this only marginally influences the separation of the taxa in the analysis. A false marker is a single character separating taxa from each other and expressing variability within the taxa, which is not reflected in other characters of the taxa. If such a false marker is excluded from a principal component analysis, the taxa are randomly distributed. In this study the characters tetradecanoic acid $\left(\mathrm{C}_{14: 0}\right)$, 13-methyltetradecanoic acid $\left(\mathrm{C}_{\text {iso15 }}\right)$, 12-methyltetradecanoic acid $\left(\mathrm{C}_{\text {ante15 }}\right)$, 3-hydroxy-13-methyltetradecanoic acid $\left(\mathrm{C}_{3-\mathrm{OH}-\mathrm{iso15}}\right)$, 3-hydroxy15-methylhexadecanoic acid $\left(\mathrm{C}_{3-\mathrm{OH} \text {-isol7 }}\right)$, and the quotient between the amounts of 13-methyltetradecanoic acid and 12-methyltetradecanoic acid seemed to be true markers in separating the genus Porphyromonas from the Bacteroides spp. strains. However, due to the few strains of Porphyromonas spp. and Bacteroides spp. tested, it will be necessary to confirm this conclusion by investigating a larger set of strains.

Our data support the creation of the new genus Porphyromonas $(20)$. In this paper we also describe cellular hydroxylated fatty acids of Porphyromonas spp., confirm previous observations of Mayberry et al. (13) and Lambe et al. (9), and show that cellular hydroxylated fatty acids are useful taxonomic markers.

\section{ACKNOWLEDGMENTS}

Financial support was provided by the Swedish Medical Research Council (projects 4977 and 6270).

\section{LITERATURE CITED}

1. Brondz, I., and I. Olsen. 1984. Determination of acids in whole lipopolysaccharide and in free lipid A from Actinobacillus actinomycetemcomitans and Haemophilus aphrophilus. J. Chromatogr. 308:19-29.

2. Brondz, I., and I. Olsen. 1984. Whole-cell methanolysis as a rapid method for differentiation between Actinobacillus actinomycetemcomitans and Haemophilus aphrophilus. J. Chromatogr. 311:347-353.

3. Brondz, I., and I. Olsen. 1985. Sugar composition of lipopolysaccharide from Haemophilus paraphrophilus. J. Chromatogr. 345:119-124.

4. Caroff, M., S. Lebbar, and L. Szabo. 1987. Do endotoxins devoid of 3-deoxy-D-manno-2-octulosonic acid exist? Biochem. Biophys. Res. Commun. 143:845-847.

5. Holdeman, L. V., P. Cato, and W. E. C. Moore (ed.). 1977. Anaerobic laboratory manual, 4th ed. Virginia Polytechnic Institute and State University, Blacksburg.

6. Johne, B., I. Olsen, and K. Bryn. 1988. Fatty acids and sugars in lipopolysaccharides from Bacteroides intermedius, Bacteroides gingivalis, and Bacteroides loescheii. Oral Microbiol. Immunol. 3:22-27.

7. Koga, T., T. Nishihara, T. Fujiwara, T. Nisizawa, N. Okahashi, T. Noguchi, and S. Hamada. 1985. Biochemical and immunobiological properties of lipopolysaccharide (LPS) from Bacteroides gingivalis and comparison with LPS from Escherichia coli. Infect. Immun. 47:638-647.

8. Kumada, H., K. Watanabe, T. Umemoto, Y. Haishima, S. Kondo, and K. Hisatsune. 1988. Occurrence of $O$-phosphorylated 2-keto-3-deoxyoctonate in the lipopolysaccharide of $\mathrm{BaC}$ teroides gingivalis. FEMS Microbiol. Lett. 51:77-80.

9. Lambe, D. W., Jr., K. P. Ferguson, and W. R. Mayberry. 1982. Characterization of Bacteroides gingivalis by direct fluorescent antibody staining and cellular fatty acid profiles. Can. J. Microbiol. 28:367-374.

10. Mansheim, B. J., A. B. Onderdonk, and D. L. Kasper. 1978. Immunochemical and biologic studies of the lipopolysaccharide of Bacteroides melaninogenicus subspecies asaccharolyticus. J. Immunol. 120:72-78.

11. Mardia, K. V., J. T. Kent, and J. M. Bibby. 1979. Multivariate analysis. Academic Press, Inc., New York.

12. Mashimo, J., M. Yoshida, K. Ikeuchi, S. Hata, S. Arata, N. Kasai, K. Okuda, and I. Takazoe. 1985. Fatty acid composition and Shwartzman activity of lipopolysaccharides from oral bacteria. Microbiol. Immunol. 29:395-403.

13. Mayberry, W. R., D. W. Lambe, Jr., and K. P. Ferguson. 1982. Identification of Bacteroides species by cellular fatty acid profiles. Int. J. Syst. Bacteriol. 32:21-27.

14. Mayrand, D., and S. C. Holt. 1988. Biology of asaccharolytic black-pigmented Bacteroides species. Microbiol. Rev. 52:134 152.

15. Millar, S. J., E. G. Goldstein, M. J. Levine, and E. Hausmann. 1986. Modulation of bone metabolism by two chemically distinct lipopolysaccharide fractions from Bacteroides gingivalis. Infect. Immun. 51:302-306.

16. Miyagawa, E., R. Azuma, and T. Suto. 1979. Cellular fatty acid composition in gram-negative obligately anaerobic rods. J. Gen. Appl. Microbiol. 25:41-51.

17. Nair, B. C., W. R. Mayberry, R. Dziak, P. B. Chen, M. J. Levine, and E. Hausmann. 1983. Biological effects of a purified lipopolysaccharide from Bacteroides gingivalis. J. Periodontal Res. 18:40-49.

18. Rizza, V., A. N. Tucker, and D. C. White. 1970. Lipids of Bacteroides melaninogenicus. J. Bacteriol. 101:84-91.

19. Shah, H. N., and M. D. Collins. 1980. Fatty acid and isoprenoid quinone composition in the classification of Bacteroides melaninogenicus and related taxa. J. Appl. Bacteriol. 48:75-87. 
20. Shah, H. N., and M. D. Collins. 1988. Proposal for reclassification of Bacteroides asaccharolyticus, Bacteroides gingivalis, and Bacteroides endodontalis in a new genus, Porphyromonas. Int. J. Syst. Bacteriol. 38:128-131.

21. Van Steenbergen, T. J. M., A. J. Van Winkelhoff, D. Mayrand, D. Grenier, and J. De Graaff. 1984. Bacteroides endodontalis sp. nov., an asaccharolytic black-pigmented Bacteroides spe- cies from infected dental root canals. Int. J. Syst. Bacteriol. 34:118-120.

22. Wold, S. 1978. Cross-validatory estimation of the number of components in factor principal components models. Technometrics 20:397-400.

23. Wold, S., K. Esbendsen, and P. Geladi. 1987. Principal component analysis. Chemometr. Intellig. Lab. Syst. 2:37-52. 